\title{
AUTOTRANSPLANTE RENAL LAPAROSCÓPICO EXPERIMENTAL
}

\author{
A. AGUILERA BAZÁN*, S. MURILLO**, J. BENITO DE LA VÍBORA**, \\ J. CISNEROS LEDO*, J. DE LA PEÑA BARTHEL*
}

*Servicio de Urología. Hospital Universitario La Paz. Madrid.

**Facultad de Veterinaria. Universidad Complutense. Madrid.

Actas Urol Esp. 28 (1): 27-31, 2004

\section{RESUMEN}

AUTOTRANSPLANTE RENAL LAPAROSCÓPICO EXPERIMENTAL

La cirugía laparoscópica requiere un largo proceso de aprendizaje en el que se va aumentando progresivamente el grado de complejidad. La técnica aquí presentada se ha realizado buscando un modelo experimental que nos permita realizar sutura vascular laparoscópica, de manera que el objetivo inmediato no es conseguir un autotransplante funcionante, sino realizar con éxito anastomosis vasculares. Presentamos nuestra experiencia en los dos primeros casos realizados.

PALABRAS CLAVE: Autotransplante renal. Entrenamiento laparoscópico. Laparoscopia vascular. Irrigación.

\section{ABSTRACT}

\section{EXPERIMENTAL LAPAROSCOPIC RENAL AUTOGRAFT}

Laparoscopic surgery requires a long training period of time in which the complexity of the training is increased. The technique presented in this paper has been developed in order to find an experimental model that allows us to improve the learning of the vascular suture. Our main goal was to evaluate this technique as an experimental model for the vascular anastomosis, not to obtain a functional autotransplant. In this regard, here we summarize our experience during the first two cases performed.

KEY WORDS: Renal autograft. Training laparoscopy. Vascular laparoscopy. Irrigation.

$L^{a}$ a cirugía laparoscópica urológica se encuentra en franco crecimiento y cada vez son más los centros interesados en realizar todo tipo de intervenciones por esta técnica. Sin embargo, a nadie se le escapa el hecho de que se trata de una técnica difícil de asimilar y con una curva de aprendizaje prolongada que requiere una gran inversión tanto económica por parte del Centro que la va a implantar (instrumental, aprendizaje en hospitales punteros) como de tiempo por parte de los cirujanos y ayudantes ${ }^{1}$.
Los principales retos que el cirujano se encuentra al iniciarse en esta técnica son varios: pérdida de la visión tridimensional (superada en algunos casos con la llegada de nuevas tecnologías), pérdida del tacto (la laparoscopia asistida por la mano suple esta dificultad), pérdida de la coordinación entre el ojo y la mano (vamos a operar sin ver nuestras manos), limitaciones tecnológicas del instrumental desarrollado que todavía está en plena evolución y además habría que añadir que supone no poder realizar muchas de 
las maniobras aprendidas en la cirugía convencional, lo que mermará nuestra capacidad ${ }^{2}$. Todo esto hace que el aprendizaje sea laborioso y, si bien no hay una pauta única a seguir, es cierto que el sentido común marca lo que debe ser este proceso.

El desarrollo de la habilidad quirúrgica en laparoscopia debe pasar necesariamente por un entrenamiento previo y exhaustivo en los sistemas de simulación quirúrgica (endotrainer), que permitirán adquirir familiaridad con el instrumental y con la diferente visión espacial que requiere esta técnica ${ }^{3}$. El siguiente paso debe ser el trabajo experimental en modelos animales vivos en el que aumentaremos la habilidad en la producción del neumoperitoneo, colocación de trócares, disección de tejidos y desarrollo de la técnica quirúrgica propiamente dicha.

En este sentido, una de las técnicas que más pueden aportar al entrenamiento en laparoscopia es el autotransplante renal. Aunque esta cirugía no es muy frecuente en la práctica diaria debido a la escasez de casos, su realización requiere un gran dominio de la disección y sutura vascular, aspectos que en laparoscopia adquieren una extrema dificultad ${ }^{4}$. El hecho de realizar autotransplantes renales exitosos implica una habilidad lo suficientemente desarrollada como para poder abordar cualquier otro tipo de cirugía urológica con garantía.

De esta manera, el objetivo de este trabajo es referir la experiencia de nuestro Servicio en el uso de esta cirugía como sistema de entrenamiento en laparoscopia. El objetivo final no es la consecución de un autotransplante funcionante, sino la realización de una sutura vascular exitosa como sistema de aprendizaje de técnica laparoscópica. Para ello, hemos elegido el cerdo como modelo de animal experimental.

\section{MATERIAL Y MÉTODOS}

Diseño experimental

Se sometió a cirugía laparoscópica a 2 hembras de cerdo (suidos híbridos de las razas LargeWhite y Landrace), de 15 y $17 \mathrm{~kg}$, respectivamente. Los animales estuvieron entre $24-48 \mathrm{~h}$ en un área preoperatoria. Tuvieron un periodo de ayuno de $12 \mathrm{~h}$, pero permanecieron con acceso libre al agua.

\section{Cuidados animales}

El Servicio de Cirugía Experimental del Hospital Universitario La Paz revisó y aprobó los protocolos del experimento, tal como exigen los Dictámenes Europeos para la protección de los animales utilizados con fines científicos y experimentales (86/609/EEC).

\section{Protocolos anestésicos}

La premedicación consistió en la administración intramuscular de ketamina $(20 \mathrm{mg} / \mathrm{kg})$ (Ketolar ${ }^{\circledR}$, Parke-Davis), diacepan (1 $\left.\mathrm{mg} / \mathrm{kg}\right)$ (Valium ${ }^{\circledR} 10$, Roche) y atropina $(0,05 \mathrm{mg} / \mathrm{kg})$ (Atropina ${ }^{\circledR}$, Bräun) conjunta en la misma jeringa. Cuando los animales fueron trasladados al quirófano, se procedió a la preoxigenación por medio de una mascarilla de Hall (5-6 1/min) durante un tiempo mínimo de cinco minutos. La inducción se realizó por medio de isofluorano (Forane ${ }^{\circledR}$, Abbott Laboratories, S.A., Madrid) al 5\% hasta alcanzar un nivel anestésico suficiente para permitir la intubación endotraqueal y la canalización de una vía venosa permeable. Después de aplicar presión en la base del pabellón auricular para distender la vena auricular, canalizamos esta vía venosa utilizando un catéter de $20 \mathrm{G}$ de calibre y $2,5 \mathrm{~cm}$ de longitud, con el fin de tener una vía permeable para la administración de fármacos y el oportuno protocolo de mantenimiento de fluidos. Comenzó entonces la administración de Ringer Lactato $(10 \mathrm{ml} / \mathrm{kg} / \mathrm{h})$. Se fijó la vía con esparadrapo adhesivo pegado alrededor de la oreja.

La intubación endotraqueal se realizó con el animal en posición de decúbito prono. Se utilizó un tubo endotraqueal de $7 \mathrm{~mm}$ d.i. Una vez finalizada la intubación, se instauró ventilación mecánica empleando para ello un ventilador (Anaesthesia Ventilator 7700, Ohmeda, England) con una fracción inspirada (Fi) de oxígeno del $100 \%$, una presión inspiratoria pico máxima (PIP) de $20 \mathrm{~cm}$ de $\mathrm{H}_{2} \mathrm{O}$, un volumen corriente $\left(\mathrm{V}_{\mathrm{T}}\right)$ de $10-15 \mathrm{ml} / \mathrm{kg}$, y una presión positiva intermitente (PEEP) de $5 \mathrm{~cm}$ de $\mathrm{H}_{2} \mathrm{O}$, para mantener la normocarmia (35-45 mm Hg). El mantenimiento anestésico se realizó con isofluorano $(2-2,5 \%)$. La concentración alveolar mínima (CAM) de isofluorano para el suido es 1,45$2,04 \%$. 
La preanalgesia consistió en la administración de un fármaco opiáceo, petidina $(2 \mathrm{mg} / \mathrm{kg} \mathrm{IV})$ (Dolantina ${ }^{\circledR}$, Bayer), en combinación con un antiinflamatorio no esteroideo (Aine), ketorolaco (0,5 $\mathrm{mg} / \mathrm{kg}$ IV) (Droal ${ }^{\circledR}$, Laboratorios Vita, S.A.).

\section{Monitorización}

Los animales se monitorizaron de forma continuada durante toda la anestesia. Esta monitorización consistió en capnografía, oximetría, concentraciones espiradas de oxígeno e isofluorano, pulsioximetría (Capnomac Ultima, Datex-Ohmeda. Helsinki. Finland), electrocardiograma (ECG) y presión sanguínea no invasiva (PSNI) (Cardiocap II, Datex-Ohmeda. Helsinki. Finland). La pinza de pulsioximetría se puso en la lengua. Un sensor de temperatura fue colocado en esófago.

\section{Técnica quirúrgica}

Con el animal colocado en decúbito lateral izquierdo (inicialmente elegimos el riñón derecho ya que el izquierdo tiene más probabilidad de tener más de una vena, lo que dificultaría enormemente la anastomosis venosa) realizamos el neumoperitoneo con aguja de Veress pinchando a nivel umbilical. Se trabajó con presiones intraabdominales de 12-13 mm Hg. Procedemos a la colocación de los trócares; en esta primera fase colocamos inicialmente tres trócares (dos de 10 $\mathrm{mm}$, uno para la cámara y otro para la mano derecha y uno de $5 \mathrm{~mm}$ para la mano izquierda) dispuestos en función de la posición del riñón del animal, buscando que la triangulación entre los tres elementos sea la adecuada. Comenzamos la disección abriendo el peritoneo por el polo inferior renal hasta identificar el uréter que nos llevará al hilio renal, si bien esta maniobra no es necesaria en estos animales dada la escasa presencia de grasa. Es importante no liberar la cara posterior renal, ya que de esta manera el riñón se encontrará más fijo y ésto nos facilitará la posterior canulación de la arteria renal. Una vez disecado el hilio renal con la máxima longitud de arteria y vena renal, se procede al clampaje de los mismos con clips laparoscópicos (animales de este tamaño no requieren más de un clip y la vena generalmente no requiere el uso de Endo GIA). A continuación, con tijera laparoscópica recta se procede a hacer una pequeña apertura en la cara anterior de la arteria renal que nos permitirá canularla para realizar la irrigación del riñón. Esta irrigación la realizaremos con un Abbocath del 24 al que le conectaremos el sistema de irrigación y procederemos a la instilación de Eurocollins ${ }^{5}$, previamente enfriado en nevera. El Abbocath con la conexión de irrigación lo introduciremos a través de un cuarto puerto de $10 \mathrm{~mm}$ colocado entre los puertos de nuestras manos. Se canula la arteria renal y se comienza a irrigar el riñón, mientras realizamos la apertura de la vena renal previamente clipada y referenciamos su luz con un Prolene de 7/0. La irrigación durará aproximadamente 15-20 minutos, hasta que comience a salir por la vena líquido de irrigación. Cortamos la arteria y la espatulamos.

En la siguiente fase se realiza la disección de vasos iliacos (común y externa) y se dejan preparados para el clampaje previo a la anastomosis. En este momento se termina de disecar todo el riñón así como el uréter para permitirnos su descenso y rotación hasta afrontar el hilio renal a los vasos iliacos. Para el clampaje vascular se pueden emplear pinzas vasculares laparoscópicas (Bulldogs) o, en caso de no disponer de ellas, utilizar vessel-loop con un clip laparoscópico que haga la función de fruncir el vessel-loop al vaso.

La fase de anastomosis vascular es probablemente la más compleja y la que requiere mayores recursos. Colocamos un nuevo trócar de $5 \mathrm{~mm}$ en la zona inguinal del animal, justo delante de la pata trasera, para la mano izquierda. Comenzamos clampando solamente la vena iliaca externa; es el momento de administrar intravenosamente heparina a dosis de $1 \mathrm{ml} / \mathrm{kg}$. Abrimos la vena con un corte transversal realizado con pinzas rectas, teniendo en cuenta que se trata de vasos de aproximadamente 3-4 mm de diámetro. Identificamos la vena renal, que se suele retraer, gracias al punto de referencia dado previamente. La anastomosis vascular la vamos a realizar con dos suturas continuas de Prolene 7/0 (es conveniente evitar la aguja Visi Black ya que es más frágil y no es infrecuente que se rompa). Comenzaremos con la cara posterior de la vena, pasando la sutura por la cara interna. A continuación daremos la sutura continua de la cara anterior. Los primeros puntos son los más complejos ya que la vena renal se encuentra completamente 
retraída. La labor del ayudante será la de mantener con una pinza de agarre la vena renal próxima a la iliaca. Una vez terminada la sutura, se desclampa la vena iliaca. En ninguno de los casos realizados se observó fuga de sangre por la anastomosis. El siguiente paso es clampar la arteria iliaca externa de la misma manera que la vena. La apertura se realiza de forma similar, dando un corte con tijera recta en la cara anterior. Se procede a la anastomosis arterial con dos suturas continuas de Prolene 7/0, empezando por la cara posterior y pasando la sutura por dentro. Finalmente se desclampa la arteria iliaca. Tampoco aquí se observó fuga tras el desclampaje.

Una vez cerrados los trayectos de los trócares se despierta al animal y se le mantiene vivo para proceder a su sacrificio a los 5 días, analizándose entonces en Anatomía Patológica los riñones transplantados.

\section{RESULTADOS}

Esta cirugía ha sido realizada por completo en dos animales, con un tiempo quirúrgico total de 8 horas cada una. El tiempo de isquemia caliente (desde el clampaje hiliar hasta la canulación de la arteria) fue de 5 minutos en el primer caso y 5,30 minutos en el segundo caso.

La vena iliaca externa estuvo clampada 3 horas en ambos casos. La diferencia estuvo en el clampaje de la arteria. En el primer caso se clampó al mismo tiempo que la vena y el clampaje total de la arteria fue de 4 horas (la sutura se realizó en aproximadamente 1 hora), produciéndose una parálisis de la extremidad inferior cuando se despertó al animal, que prácticamente no llegó a recuperar en el post-operatorio. En el segundo caso se varió la técnica y se realizó primero el clampaje y la anastomosis venosa, que es la más laboriosa (3 horas), sin clampar la arteria iliaca. Posteriormente se realizó la anastomosis arterial en 1 hora y 15 minutos. En el post-operatorio el animal movía con normalidad las extremidades inferiores.

Al sacrificar a los animales a los 5 días, el que tenía dificultad para movilizar la extremidad trasera derecha presentaba una trombosis de la arteria iliaca externa y de la arteria renal, habiéndose perdido el implante. En el segundo caso la arteria iliaca y renal presentaban latido y flujo.

\section{DISCUSIÓN}

Como se indicó en la introducción, el objetivo principal en esta cirugía era la evaluación del autotransplante renal como modelo de aprendizaje laparoscópico, técnica que nos permite añadir la sutura vascular a nuestros recursos. A las dificultades propias de la sutura vascular hay que añadir dos factores que han aumentado de forma considerable la dificultad de la cirugía en este estudio, como son el tamaño del animal y el del instrumental y que deben ser tenidos en cuenta a la hora de realizar cualquier cirugía por vía laparoscópica.

El tamaño del animal, hembras de entre $15 \mathrm{y}$ $17 \mathrm{~kg}$, resultó pequeño, lo que se tradujo en la presencia de vasos renales e iliacos de reducido diámetro que dificultaron en gran medida la sutura vascular. Animales más grandes deberían facilitar la técnica sobre todo al comienzo del aprendizaje. El hecho de elegir hembras como modelo experimental en este caso no es imprescindible, aunque nos permite el entrenamiento en otras cirugías más sencillas que pueden ser realizadas posteriormente en el animal o en su cadáver y que requieren la presencia de útero, como la sutura vésico-ureteral en el que empleamos el útero como modelo de uréter. Así mismo, el instrumental empleado deberá ajustarse al tamaño de la sutura empleada ya que su nocorrespondencia ocasiona deformaciones en la aguja. Aún así, nosotros fuimos capaces de realizar la técnica con porta-agujas de $5 \mathrm{~mm}$, en lugar de los $3 \mathrm{~mm}$ que deberían haber sido empleados en animales de ese peso.

Otro aspecto a tener en cuenta es el sistema de enfriamiento para disminuir el tiempo de isquemia caliente. En nuestro caso hemos empleado la canulación de la arteria renal y su posterior irrigación con Eurocollins previamente enfriado a $4^{\circ} \mathrm{C}$, aunque los resultados obtenidos han sido sastisfactorios en cuanto a la realización de las anastomosis vasculares, ya que no hubo sangrados después del desclampaje y se observaba latido, la función renal se vio seguramente dañada por el sistema de enfriamiento usado. Para mantener la funcionalidad del riñón a lo largo del transplante se debería añadir al sistema aquí empleado una fuente de enfriamiento externo, consistente en una bolsa de extracción de 
pieza de laparoscopia con hielo muy picado que se pudiera introducir a través del trócar o con la instilación de suero a $4^{\circ}$ en la que se introduciría el riñón. Sin embargo, al no ser el objetivo del trabajo la consecución de un autotransplante funcionante, se optó por el otro sistema que disminuye el tiempo quirúrgico en gran medida.

Posteriormente a estos dos autotransplantes se han realizado otras técnicas laparoscópicas en nuestro Quirófano Experimental (neovejiga intestinal, anastomosis vésico-uretral) que ya se venían realizando con anterioridad, notándose un notable incremento en la rapidez y seguridad de la técnica quirúrgica, que se traducen en una disminución efectiva de los tiempos quirúrgicos.

Indudablemente, esta cirugía no es la técnica más adecuada para iniciarse en laparoscopia. Ha sido realizada en nuestro Servicio tras un largo proceso de aprendizaje que comenzó con técnicas básicas (manejo de endotrainer, ejercicios de sutura) que van ganando en complejidad de forma escalonada (nefrectomía, sutura vesical, disección vascular, nefrectomía parcial, anastomosis vésico-ure- teral, colgajo vesical, anastomosis vésico-uretral, neovejiga intestinal), pero ha resultado ser de gran ayuda a la hora de adquirir habilidad en el manejo del instrumental y de la visión espacial, así como en el ejercicio de disección vascular.

\section{REFERENCIAS}

1. SAKTI DAS.: Laparoscopia urológica avanzada. Clínicas Urológicas de Norteamérica 2001; 1: 5-14.

2. GÜNTER JANETSCHEK, JENS RASSWEILER.: Cirugía laparoscópica en urología. Ed. Masson 1998; 6: 55-64.

3. TOPEL HC.: Endoscopio suturing and knot tying manual. Ethicon, Hamburg 1991.

4. HUNTER JG, SACKIER JM.: Training for minimally invasive surgery. Mc Graw-Hill. New York 1993.

5. JOHN A. LIBERTINO.: Campbell's Urology $7^{\text {a }} \mathrm{Ed}$. Walsh, Retik, Vaughan, Wein. Ed. Saunders; 12: 460-489.

\author{
Dr. A. Aguilera Bazán \\ Servicio de Urología. Hospital La Paz \\ Po de la Castellana, 261 \\ 28046 Madrid
}

(Trabajo recibido el 1 septiembre 2003)

\section{COMENTARIO EDITORIAL}

El desarrollo de las técnicas laparoscópicas ha revolucionado la cirugía urológica en la última década. En particular en el ámbito del trasplante renal la menor invasividad de la nefrectomía laparoscópica ha incrementado el número de trasplantes renales con donante vivo, incluso en un país como España en el que la alta donación de cadáver reducía clásicamente la tasa de trasplante renal con donante vivo por debajo del $1 \%$.

Sin embargo, la utilidad de la laparoscopia en la cirugía renovascular parece mucho más controvertida. De hecho, tan sólo existe una referencia en la clínica de reparación laparoscópica de un aneurisma de arteria renal, si bien es cierto que en manos de I. Gill ${ }^{1}$, una de las máximas autoridades en laparoscopia urológica y pionero en muchas técnicas quirúrgicas desde el ámbito de la laparoscopia.

Existe una referencia experimental previa de autotrasplante renal en cerdo $^{2}$, igualmente del Grupo de la Cleveland Clinic, que reconoce la dificultad técnica, la larga duración y el hecho de que la realización en la práctica clínica, aunque quizá no esté muy lejana, no es una realidad en la actualidad.

Por ello, aunque el presente trabajo incluye solamente 2 animales, constituye la primera referencia de esta técnica en la literatura española y describe de modo detallado los pasos que incluye la misma: nefrectomía, perfusión fría in situ y técnica de anastomosis vascular, describiendo las dificultades que cada uno de estos pasos conlleva. En cualquier caso, la finalización de este tipo de cirugía laparoscópica por parte de los autores merece la felicitación por su adiestramiento en el ámbito experimental de la laparoscopia urológica.

\section{REFERENCIAS}

1. GILL IS, MURPHY DP, HSU T, et al: Laparoscopic repair of renal artery aneurysm. J Urol 2001; 166: 202-205.

2. KAOUK H, SUNG GT, CILL IS: Laparoscopic renal autotransplantation. J Urol 2001; 165: 352

Fdo.: F.J. Burgos Revilla Hospital Ramón y Cajal. Universidad de Alcalá. Madrid 